\title{
Kann die klinische Wirksamkeit der Aerosol-Therapie mit Budesonid durch Inhalation unter Belastung verbessert werden?
}

\author{
Ann Kristin Barton', Henrike Heinemann', Roswitha Merle² und Heidrun Gehlen' \\ 1 Klinik für Pferde, Allg. Chirurgie und Radiologie der FU Berlin \\ 2 Institut für Veterinär-Epidemiologie und Biometrie der FU Berlin
}

\begin{abstract}
Zusammenfassung: Recurrent airway obstruction (RAO) und Inflammatory airway disease (IAD) stellen häufige chronische Lungenerkrankungen bei Pferden in Stallhaltung dar. In den vergangenen Jahren erfreut sich die Inhalation mit Glukokortikoiden zunehmender Beliebtheit in der Behandlung dieser Patienten. Ziel dieser Studie war es, zu untersuchen, ob die klinische Wirksamkeit der Aerosoltherapie durch die Inhalation unter Belastung mittels mobiler Inhalationsmasken verbessert werden kann. Mittels eines Scoresystems wurden 12 vorberichtlich an RAO bzw. IAD erkrankte Pferde umfassend klinisch untersucht (klinische Untersuchung, arterielle Blutgasanalyse, Bronchoskopie mit bronchoalveolärer Lavage (BAL), Zytologie der BAL-Flüssigkeit) und in die Gruppen RAO und IAD unterteilt. Danach erfolgte eine 10-tägige

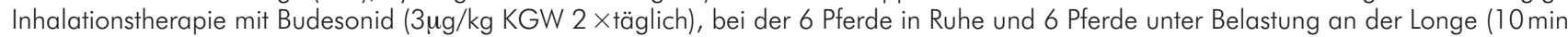
Schritt, $10 \mathrm{~min}$ Trab und $5 \mathrm{~min}$ Galopp, $10 \mathrm{~min}$ Schritt) behandelt wurden, wobei das Inhalationsgerät mit Beginn der Trabphase gestartet wurde. Danach erfolgten erneute klinische und weiterführende Untersuchungen analog der Eingangsuntersuchung. Die Inhalationstherapie führte bei allen Probanden zu einer Verbesserung der klinischen Symptomatik. Es zeigte sich eine signifikante Reduktion des klinischen Scores in der Gesamtpopulation ( $p=0,002$ ) und innerhalb der Subgruppen (Inhalation in Ruhe vs. Belastung), nicht jedoch in allen Einzelparametern wie z.B. dem Prozentanteil der neutrophilen Granulozyten. Im Gruppenvergleich ergaben sich ebenfalls keine signifikanten Unterschiede. Zusammenfassend erscheint die inhalative Verabreichung somit auch bei RAO in Exazerbation empfehlenswert. Die Aerosoltherapie unter Belastung führte jedoch nicht zu einer signifikant verbesserten Wirksamkeit.
\end{abstract}

Schlüsselwörter: Recurrent Airway Obstruction / Inhalation / Aerosoltherapie / Budesonid / Bewegung / Pferd / Pulmologie

\section{Clinical effect of budesonide aerosol therapy not augmented by inhalation under exercise}

Recurrent airway obstruction (RAO) and Inflammatory airway disease (IAD) are common respiratory diseases in stabled horses. In recent years, inhalation of glucocorticoids has become a popular therapeutic approach. We hypothesized that the success of therapy might be augmented, if aerosol therapy is performed under exercise by using mobile inhalation devices. Using a scoring system, 12 horses with a history of RAO or IAD were examined (clinical examination, arterial blood gas analysis, bronchoscopy and bronchoalveolar lavage cytology) to confirm the diagnosis and classify respiratory disease as mild to moderate (IAD or RAO in remission) or severe (RAO in exacerbation). Horses received aerosol therapy with budesonide $(3 \mu \mathrm{g} / \mathrm{kg}$ BDW twice daily). 6 animals were inhaled at rest and the remaining 6 during lunging exercise (10 min walk, $10 \mathrm{~min}$ trot, $5 \mathrm{~min}$ canter, $10 \mathrm{~min}$ walk). In the later, the inhalation device was started with the beginning of trotting. Afterwards, the clinical examination was repeated and differences in clinical scores and single parameters before and after therapy compared between the two groups. Aerosol therapy led to clinical improvement in all 12 horses. The clinical score was significantly reduced in the overall population $(p=0.002)$ and in the subgroups, while no significant differences were found in single parameters evaluated. There were no significant differences in treatment success between the two inhalation groups. In conclusion, aerosol therapy can be recommended even in severe cases of RAO. Aerosol therapy under exercise does not augment the clinical effect of budesonide, but may ease handling of affected horses during inhalation.

Keywords: Recurrent airway obstruction / aerosol therapy / inhalation / budesonide / exercise / horse / pulmology

Zitation: Barton A. K., Heinemann H., Merle R., Gehlen G. (2016) Kann die klinische Wirksamkeit der Aerosol-Therapie mit Budesonid durch Inhalation unter Belastung verbessert werden? Pferdeheilkunde 32, 119-123

Korrespondenz: Ann Kristin Barton, Klinik für Pferde, Allg. Chirurgie und Radiologie der FU Berlin, Oertzenweg 19b, 14163 Berlin; Email: Ann-Kristin.Barton@fu-berlin.de

\section{Einleitung}

Die Recurrent airway obstruction (RAO) ist die häufigste chronische Lungenerkrankung beim Pferd in Stallhaltung mit einer aktuellen Prävalenz von ca. $14 \%$ in der nördlichen Hemisphere (Pirie 2014). Die Prävalenz der Inflammatory Airway Disease (IAD), welche als mögliche Vorläufererkrankung bzw. mildere Form der RAO diskutiert wird (Coëtil et al. 2007; Leclere und Lavoie 2014), ist ebenfalls eine sehr häufige Erkrankung bei Sportpferden. Zur Behandlung stehen verschiedene Medikamente zur Verfügung, die systemisch (oral, i.m., i.v.) oder per Inhalation verabreicht werden können. Dabei hat die Inha- lationstherapie einige Vorteile gegenüber der systemischen Therapie, auch wenn es aktuell noch kein für Pferde zugelassenes Präparat gibt (Niedermaier und Gehlen 2009). Durch die lokale Applikation und Wirkung der Medikamente am Bestimmungsort werden systemische Nebenwirkungen weitestgehend vermieden (Duvivier et al. 1997, Dauvillier et al. $2011)$. Darüber hinaus kann die Dosierung der angewandten Medikamente z.T. erheblich reduziert und die Arzneimittelsicherheit damit erhöht werden. Mit den relativ preiswerten Inhalationshilfen für Dosieraerosole ist der Einstieg in die Inhalationstherapie darüber hinaus inzwischen deutlich preiswerter geworden. Verschiedene Autoren haben die klinische Wirk- 
samkeit der Inhalation mit Düsen- oder Ultraschallverneblern beschrieben (Robinson et al. 1993, Votion et al. 1997, Hofman et al. 1997, 2008) und zahlreiche Studien zur Wirksamkeit der Inhalation mit Dosieraerosolen und Trockenpulverinhalatoren liegen vor (Ammann et al. 1998, Derksen et al. 1999, Rush et al. 2000, Kampmann et al. 2001, Covëtil et al. 2005, 2006, Laan et al. 2006). In den letzten Jahren sind außerdem Systeme auf den Markt gekommen, die eine Inhalation unter Belastung, z.B. an der Longe oder in der Führanlage ermöglichen. Eine pharmakologische Bronchospasmolyse, die zusätzliche Medikamentenkosten verursacht, kann so im Langzeit-Management, gegebenenfalls umgangen werden. Unter Belastung steigt der Sympathikotonus an, was zu einer Bronchodilatation führt. Außerdem werden Atemfrequenz und -tiefe und somit das Atemminutenvolumen gesteigert. Daher wird möglicherweise mehr Wirkstoff aufgenommen, tiefere Anteile des Bronchialbaums erreicht und die klinische Wirksamkeit der Präparate verbessert. Ziel dieser Studie war es deshalb, den Einfluss einer Inhalation in Ruhe mit einer Inhalation unter Belastung an 12 Pferden mit RAO und IAD vergleichend zu untersuchen.

\section{Material und Methoden}

\section{Gruppeneinteilung}

Die Untersuchungen erfolgten an 12 Pferden, bei denen seit mehreren Jahren eine chronische Lungenerkrankung bekannt war (Tabelle 1). Alle Pferde waren laut Besitzerangaben bereits im Heimatstall haltungsoptimiert (staubarme Einstreu, nasses Heu oder Silage, z.T. Offenstallhaltung), die durchgeführten Maßnahmen waren jedoch z.T. sehr heterogen. Im Rahmen einer klinischen Eingangsuntersuchung wurde die Diagnose zunächst als RAO in Exazerbation oder IAD bzw. $\mathrm{RAO}$ in Remission bestätigt. Anhand des Vorberichtes war eine sichere Differenzierung zwischen IAD und RAO in Remission nicht in allen Fällen möglich und eine Provokationsprobe mit schimmeligem Heu wurde im Rahmen dieser Studie nicht durchgeführt.

Danach erfolgte eine zufällige Einteilung der Probanden in zwei Gruppen. Eine Gruppe wurde zweimal täglich in Ruhe inhaliert, die andere unter Belastung an der Longe. Danach erfolgte eine zweite Untersuchung analog der Eingangsuntersuchung.

\section{Eingangsuntersuchung}

Bei allen Pferden wurden an drei aufeinander folgenden Tagen (Tag 1-3) klinische Allgemeinuntersuchungen, spezielle klinische Untersuchungen des Atmungsapparates (Auskultation und Perkussion der Lunge) sowie arterielle Blutgasanalysen durchgeführt. Des Weiteren wurde eine Endoskopie mit bronchoalveolärer Lavage durchgeführt, um den aktuellen Erkrankungsstatus der Probanden zu ermitteln. Der Schweregrad der Erkrankung wurde mit Hilfe eines Untersuchungsscores (Ohnesorge et al. 1998) als gering- bis mittelgradig im Sinne einer IAD bzw. einer RAO im Remission oder als hochgradig im Sinne einer RAO in Exazerbation klassifiziert, wobei dieser Score insofern modifiziert wurde, als dass entsprechend aktuellen internationalen Empfehlungen (Robinson 2001) der Prozentanteil der neutrophilen Granulozyten in der bronchoaolveolären Lavageflüssigkeit anstelle des Tracheobronchialsekret erfasst wurde (siehe hierzu Tabelle 2).

\section{Inhalation}

Alle Pferde wurden über einen Zeitraum von drei Tagen (Tag 2-4) zweimal täglich für 10 min mit 0,9\%-iger $\mathrm{NaCl}$-Lösung inhaliert, um sie an das Equipment zu gewöhnen. Dieses erfolgte bei 6 Pferden in Ruhe, bei den anderen 6 unter Belastung an der Longe.

Über den Behandlungszeitraum (ab Tag 4) wurden alle Pferde staubarm in der Klinik aufgestallt (Einstreu mit Holzspänen, Fütterung von nassem $\mathrm{Heu}$ ), um gleiche Bedingungen für alle Probanden zu gewährleisten, was Haltung und Durchführung der Inhalation betraf. Die Pferde wurden über einen Zeitraum von 10 Tagen (Tag 5-14) zweimal täglich mit einem Glukokortikoid inhaliert $(3 \mu \mathrm{g} / \mathrm{kg} \mathrm{KGW}$ Budesonid, Präparat "Pulmicort", Astra Zeneca, Deutschland). Das Prä-

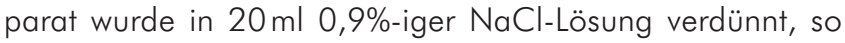
dass die Verneblungsdauer ca. 20 Minuten betrug. Die Inha-

Tab. 1 Probanden der beiden Untersuchungsgruppen, Inhalation in Ruhe vs. Inhalation unter Belastung. | Horses of both subgroups, which received inhalation therapy at rest and during lunging exercise

\begin{tabular}{|c|c|c|c|c|c|c|}
\hline Nr. & Gruppe & Rasse & Geschlecht & Größe [cm] & Gewicht [kg] & Alter [Jahre] \\
\hline 1 & Bewegung & Deutsches Warmblut & Stute & 170 & 550 & 12 \\
\hline 2 & Bewegung & American Quarter Horse & Stute & 152 & 480 & 14 \\
\hline 3 & Bewegung & Traber & Wallach & 159 & 450 & 21 \\
\hline 4 & Bewegung & Pinto & Wallach & 139 & 400 & 19 \\
\hline 5 & Bewegung & Deutsches Reitpony & Wallach & 148 & 350 & 12 \\
\hline 6 & Bewegung & Haflinger & Wallach & 149 & 450 & 21 \\
\hline 7 & Ruhe & Araber & Stute & 149 & 400 & 19 \\
\hline 8 & Ruhe & Traber & Wallach & 162 & 535 & 10 \\
\hline 9 & Ruhe & Araber & Stute & 150 & 450 & 23 \\
\hline 10 & Ruhe & Trakehner & Wallach & 166 & 600 & 14 \\
\hline 11 & Ruhe & Deutsches Warmblut & Stute & 162 & 550 & 22 \\
\hline 12 & Ruhe & Deutsches Warmblut & Stute & 163 & 500 & 18 \\
\hline
\end{tabular}


lation erfolgte bei 6 Pferden in Ruhe, bei den anderen 6 unter Belastung nach anfänglicher Aufwärmphase im Schritt (10 min Schritt, $10 \mathrm{~min}$ Trab und $5 \mathrm{~min}$ Galopp, $10 \mathrm{~min}$ Schritt), wobei das Inhalationsgerät mit Beginn der Trabphase gestartet wurde. Auch die in Ruhe inhalierten Pferde wurden zweimal täglich nach diesem Protokoll bewegt, um eine ausreichende Bewegung der Pferde zu gewährleisten, wobei jedoch ein zeitlicher Abstand von mindestens 4 Stunden zur Inhalation eingehalten wurde.

\section{Kontrolluntersuchung}

Nach Ende der Inhalationsphase erfolgte eine Nachkontrolle analog der Eingangsuntersuchung (Tag 14-16).

\section{Statistik}

Die statistische Auswertung erfolgte mit der Software SPSS (IBM SPSS Version 22). Die Daten wurden zunächst auf Normalverteilung geprüft. Da keine Normalverteilung vorlag, erfolgte die weitere statistische Auswertung mit dem Wilcoxon-Vorzeichen-Rang-Test mit einem Signifikanzniveau von $p<0,05$ für die Gesamtpopulation. Für die Auswertung der Untergruppen wurde das Signifikanzniveau aufgrund der geringen Stichprobengröße auf $p<0,1$ angehoben. Innerhalb der gepaarten Stichproben wurden die Differenzen des Gesamtscores sowie der Einzelparameter, z.B. AaDO2 oder Prozentanteil der neutrophilen Granulozyten in der BALF, vor und nach Behandlung gebildet, und es wurde mit dem Wilcoxon-Test für gepaarte Beobachtungen geprüft, ob sich die Werte vor und nach der Behandlung signifikant voneinander unterschieden. Anschließend wurden die Werte zwischen den beiden Untergruppen (Inhalation in Ruhe und unter Belastung) verglichen, um herauszufinden, ob es signifikante Unterschiede im Therapieerfolg zwischen den beiden Behandlungsmethoden gab (Wilcoxon-Test für unabhängige Stichproben).

\section{Ergebnisse}

Bei allen 12 Probanden wurde die vorberichtliche chronische Lungenerkrankung im Sinne einer RAO oder IAD bestätigt. 6 Pferde wurden als IAD/RAO in Remission und 6 Pferde als RAO in Exazerbation klassifiziert (Tabelle 3). Durch die Inhalationstherapie wurde bei allen 12 Pferden (Tabelle 3) und in der Gesamtheit der untersuchten Pferde eine hochsignifikante Reduktion des klinischen Gesamtscores $(p=0,002)$ erreicht werden. Bei den im Klinikalltag als maßgeblich bewerteten Parametern für den Gasaustausch $\mathrm{PaO}^{2}$ $(p=0,109)$ und $\operatorname{AaDO}^{2}(p=0,078)$, sowie für den als maßgeblichen Indikator für den Schweregrad der Entzündung

Tab. 2 Untersuchungsscore zur Klassifizierung des Schweregrades der chronischen Lungenerkrankung, modifiziert nach Ohnesorge et al.

(1998). Modified clinical scoring system for the evaluation of chronic respiratory disease severity according to Ohnesorge et al. (1998)

\begin{tabular}{|c|c|c|}
\hline Untersuchung & Befund & Punktzahl \\
\hline $\begin{array}{l}\text { Hustenauslösung } \\
\text { (max. } 1 \text { Punkt) }\end{array}$ & $\begin{array}{l}\text { Nicht auslösbar } \\
\text { Mehrfach auslösbar } \\
\text { Auswurf } \\
\text { Spontaner Husten }\end{array}$ & $\begin{array}{l}0 \\
1 \\
1 \\
1\end{array}$ \\
\hline $\begin{array}{l}\text { Ruhedyspnoe } \\
\text { (max. } 3 \text { Punkte) }\end{array}$ & $\begin{array}{l}\text { Abdominale Atmung } \\
\text { Verlängerte Exspiration } \\
\text { Einsinkende ICR } \\
\text { Nüsternblähen } \\
\text { Damprinne } \\
\text { Afteratmung }\end{array}$ & $\begin{array}{l}1 \\
1 \\
3 \\
3 \\
3 \\
3\end{array}$ \\
\hline $\begin{array}{l}\text { Lungenperkussion } \\
\text { (max. } 2 \text { Punkte) }\end{array}$ & $\begin{array}{l}\text { Erweiterung } 3 \text { Finger } \\
\text { Erweiterung handbreit } \\
\text { Dämpfung }\end{array}$ & $\begin{array}{l}0 \\
1 \\
2\end{array}$ \\
\hline $\begin{array}{l}\text { Lungenauskultation } \\
\text { (max. } 2 \text { Punkte) }\end{array}$ & $\begin{array}{l}\text { Rasseln } \\
\text { Knistern } \\
\text { Giemen }\end{array}$ & $\begin{array}{l}2 \\
2 \\
2\end{array}$ \\
\hline $\begin{array}{l}\text { Tracheobronchoskopie } \\
\text { (max. } 2 \text { Punkte) }\end{array}$ & $\begin{array}{l}\text { Sekret deutlich vermehrt u./o. mäßig viskös } \\
\text { Sekret hgr. vermehrt u./o. zähviskös } \\
\text { Carina tracheae deutlich verdickt }\end{array}$ & $\begin{array}{l}1 \\
2 \\
1\end{array}$ \\
\hline $\begin{array}{l}\text { BALF Zytologie } \\
\text { (max. } 3 \text { Punkte) }\end{array}$ & $\begin{array}{c}\text { Neutrophile Granulozyten < 10\% } \\
\text { Neutrophile Granulozyten 10-15\% } \\
\text { Neutrophile Granulozyten 15-25\% } \\
\text { Neutrophile Granulozyten > 25\% }\end{array}$ & $\begin{array}{l}0 \\
1 \\
2 \\
3\end{array}$ \\
\hline $\begin{array}{l}\text { Blutgasanalyse } \\
\text { (max. } 2 \text { Punkte) }\end{array}$ & $\begin{array}{l}\mathrm{AaDO}_{2} 7-14 \mathrm{mmHg} \\
\mathrm{AaDO}_{2}>14 \mathrm{mmHg}\end{array}$ & $\begin{array}{l}1 \\
2\end{array}$ \\
\hline
\end{tabular}


gesehene prozentualen Anteil der neutrophilen Granulozyten in der BALF zeigten sich keine statistisch signifikante Veränderung nach Behandlung $(p=0,423)$. Auch zwischen den beiden Therapie-Gruppen (Inhalation in Ruhe vs. Belastung) ergaben sich im klinischen Gesamtscore statistisch signifikante Unterschiede vor und nach Therapie (Inhalation in Ruhe $p=0,062$, Inhalation in Bewegung $p=0,062)$. Bei isolierter Betrachtung der Einzelparametern $\mathrm{AaDO}^{2}$ und dem Prozentsatz der neutrophilen Granulozyten war jedoch in diesen Gruppen statistisch kein signifikanter Unterschied vor und nach Behandlung. Bei dem Vergleich zwischen den beiden Therapie-Gruppen ergaben sich sowohl für den klinischen Gesamtscore ( $p=0,275)$ als auch für die Einzelparameter keine signifikanten Unterschiede zwischen Inhalation in Ruhe und unter Belastung.

Bei einer separaten Auswertung der an RAO in Exazerbation erkrankten Pferde ( $\geq 7$ Scorepunkte, $n=6$ ) wurden eine signifikante Verbesserung des Gesamtscores $(p=0,031)$ und der $\mathrm{AaDO}^{2}(p=0,062)$ festgestellt.

\section{Diskussion}

Bei allen Probanden konnte nach 10-tägiger Inhalation anhand des Gesamtscores eine signifikante Verbesserung festgestellt werden. Im Gegensatz zu der Studie von Kampmann et al. (2001), in der bereits eine klinische Verbesserung bei gering- und mittelgradig an Chronisch obstruktiver Bronchitis erkrankten Pferden beschrieben wurde, nach heutiger Terminologie weitgehend der IAD bzw. RAO in Remission entsprechend, zeigte die Betrachtung der Ergebnisse der einzelnen Pferde und der Subgruppe der hochgradig erkrankten Tiere in unserer Studie jedoch, dass auch bei den hochgradig im Sinne einer RAO in Exazerbation erkrankten Pferde ein signifikanter, im Einzelfall z.T. sogar deutlicherer Therapieerfolg zu verzeichnen war (Nr. 3, 5 und 6), als bei den gering- bis mittelgradig im Sinne einer IAD erkrankten Tieren. Aufgrund potentieller Nebenwirkungen systemisch verabreichter Glukokortikoide (Duvivier et al. 1997, Dauvillier et al. 2011) erscheint daher aus medizinischer Sicht ein Therapieversuch mit inhalativ verabreichten Wirkstoffen auch bei hochgradig erkrankten Pferden sinnvoll.

Leider stehen zurzeit noch keine zugelassenen Präparate für die inhalative Anwendung von Glukokortikoiden beim Pferd zur Verfügung, so dass nach dem Arzneimittelrecht ein Therapienotstand z.B. bei mangelhaftem Ansprechen auf eine systemische Glukokortikoidgabe oder bei Auftreten von Nebenwirkungen bei systemischer Gabe, vorliegen muss, um Glukokortikoide überhaupt inhalativ einsetzen zu dürfen. Es erscheint auf Basis der Ergebnisse unserer Studie jedoch wünschenswert, dass sich hier in Zukunft durch die Zulassung eines entsprechenden Präparates für Pferde etwas ändert.

Die therapeutische Effektivität von inhalativen Glukokortikoiden wird beeinflusst von ihrer Lipophilie, ihrer Ablagerung in der Lunge und ihrer Akkumulation im Lungengewebe, wohingegen die Eliminationsrate die systemische Wirkung determiniert. Budesonid verbleibt im Gegensatz zu anderen inhalativ zu verabreichenden Glukortikoiden aufgrund eines Veresterungsprozesses, welches die Lipophilie verstärkt, deutlich länger im Lungengewebe (O'Connel/ 2003). Dies erklärt die verlängerte klinische Wirksamkeit, obwohl die Rezeptorbindungsaffinität deutlich unter der von Beclomethason und Fluticason liegt (Pirie 2014).

Im Vergleich der Inhalationsgruppen konnten in unserer Studie keine signifikanten Unterschiede festgestellt werden. Die Hypothese, dass bei Inhalation unter Belastung durch das tiefere Einatmen des Patienten mehr Wirkstoff in die Lunge gelangt und so eine höhere Effektivität der Therapie gegeben ist, konnte somit nicht bestätigt werden. Nichtsdestotrotz kann die Anwendung einer Inhalationsmaske, die auch unter Bewegung des Pferdes eingesetzt werden kann, sicherlich vorteilhaft sein bei Tieren, die die Maske in Ruhe schlecht tolerieren. Unserer Studie zeigte, dass durch die gleichzeitige Belastung währen der Inhalation die Pferde abgelenkt waren, weniger Abwehrbewegungen vorkamen und das Inhalations-Handling so erleichtert wurde. Auch stellt eine regelmäßige, submaximale Bewegung des Pferdes

Tab. 3 Teil der Ergebnisse der klinischen und labordiagnostischen Untersuchungen vor und nach Inhalationstherapie mit Budesonid, \% PMN = Anteil der neutrophilen Granulozyten in der BALF, $\mathrm{AaDO}_{2}=$ arterio-alveoläre Druckdifferenz, hochgradig erkrankte Pferde grau unterlegt, Pferde Nr. 1-6 Inhalation in Bewegung, Nr. 7-12 Inhalation in Ruhe. Parts of the results of clinical and laboratory examination before and after inhalation therapy with budesonide, \%PMN = percentage of neutrophil granulocytes in $B A L F, A a D O_{2}=$ arterio-alveolar pressure difference, severely affected horses shimmed in grey, horses No. 1-6 inhaled during exercise, No. 7-12 at rest

\begin{tabular}{|c|c|c|c|c|c|c|}
\hline Nr. & $\%$ PMN vorher & $\mathrm{AaDO}_{2}$ vorher & Gesamtscore vorher & $\%$ PMN nachher & $\mathrm{AaDO}_{2}$ nachher & Gesamtscore nachher \\
\hline 1 & 18,8 & 0 & 4 & 2,5 & 0 & 0 \\
\hline 2 & 52 & 2,0 & 8 & 67,8 & 0 & 6 \\
\hline 3 & 87,8 & 12,2 & 8 & 34,2 & 0 & 5 \\
\hline 4 & 27,8 & 1,2 & 4 & 69,4 & 2,5 & 4 \\
\hline 5 & 43 & 0,1 & 7 & 18,8 & 0 & 4 \\
\hline 6 & 45,8 & 18,7 & 9 & 38,2 & 1 & 3 \\
\hline 7 & 47,4 & 0 & 6 & 14,4 & 0 & 4 \\
\hline 8 & 11,8 & 0 & 2 & 1,75 & 0 & 0 \\
\hline 9 & 27 & 8,0 & 7 & 34,2 & 0 & 5 \\
\hline 10 & 84,2 & 0,9 & 6 & 56 & 0 & 5 \\
\hline 11 & 9,8 & 0 & 1 & 8 & 0 & 1 \\
\hline 12 & 15 & 0 & 8 & 49,3 & 0 & 4 \\
\hline
\end{tabular}


einen wesentlichen Therapiebestandteil bei chronischen Atemwegserkrankungen dar, um die Sekretmobilisation bei diesen Patienten zu unterstützen (Fey und Ohnesorge 2009). Somit kombiniert die Belastungsinhalation zwei Therapieoptionen.

Aufgrund der Tatsache, dass diese Studie unter Klinikbedingungen an Patienten in Privatbesitz durchgeführt wurde, ergaben sich einige Schwachpunkte, die die Aussagekraft unserer Ergebnisse sicherlich beeinträchtigen. Der größte Schwachpunkt der vorliegenden Arbeit liegt in der geringen Gruppengröße. Um statistisch signifikante Unterschiede zwischen den Gruppen nachweisen zu können, müsste die Stichprobengröße geeignet erhöht werden. Eine Stichprobenberechnung ergab, dass bei einer Gruppengröße von 22 Tieren je Gruppe ein Unterschied von 1,5 im Gesamtscore zwischen den Gruppen mit einer Sicherheit 95\% festgestellt werden kann (Poissonverteilung angenommen).

Somit wären insgesamt 44 statt 12 Probanden erforderlich gewesen, dieses war aus organisatorischen Gründen leider nicht möglich, da jeder Proband insgesamt 16 Tage in der Klinik aufgestallt wurde. Diese hohe Probandenzahl könnte in einer Feldstudie (Inhalation im Heimatstall) bearbeitet werden, allerdings hätte man hier den Nachteil einer möglichen ungleichen Durchführung der Inhalation bzw. Anwendungsfehler mit dem Inhalationsequipment durch den ungeübten Besitzer. Auch die Paarbildung gleicher Schweregrade innerhalb der Subgruppen hätte die Probandenzahl erhöht, so dass auch dieses aus organisatorischen Gründen nicht möglich war. Die Ergebnisse dieser Studie sind daher als hinweisend, nicht jedoch als absolut zu betrachten.

Neben der Inhalation unter Bewegung sollte außerdem eine mögliche Verbesserung des klinischen Effektes einer Budesonidinhalation nach pharmakologischer Bronchodilatation durch die Verabreichung von Clenbuterol p.o. oder Butylscopolamid i.v. untersucht werden. Auch wenn letzteres durch die intravenöse Applikation wenig praxisrelevant erscheint, könnte durch die Verabreichung eines Sympathomimetikums bei hochgradig erkrankten Pferden eine Bronchospasmolyse erreicht werden, bei denen eine Belastung möglicherweise gar nicht möglich ist oder die aufgrund orthopädischer Erkrankungen nicht bewegt werden dürfen. Auch die gleichzeitige Gabe eines Parasympatholytikums, welches mit dem Ipratropiumbromid in inhaltiv zu verabreichender Form zur Verfügung steht, wäre denkbar. Für uns war jedoch die Inhalationstherapie unter gleichzeitiger Bewegung besonders interessant, weil so Medikamentenkosten im Langzeitmanagement betroffener Tiere eventuell vermieden werden können.

Auch die Untersuchung einer Kontrollgruppe chronisch lungenkranker Pferde, die über den gleichen Zeitraum in der Klinik staubarm aufgestallt und einen Placebo inhaliert hätte, wäre wünschenswert gewesen. Hier eine ausreichend hohe Anzahl tatsächlicher RAO oder IAD Patienten in Privatbesitz zu akquirieren, war jedoch ebenfalls nicht möglich, und hätte erneut zu einer Erhöhung der Gesamtprobandenzahl geführt. Zusammenfassend erscheint die inhalative Verabreichung von Budesonid auch bei RAO in Exazerbation erkrankten Pferden dennoch empfehlenswert. Die Aerosoltherapie unter Belastung führte jedoch in unserer Studie mit kleiner Fallzahl nicht zu einer verbesserten Wirksamkeit, konnte aber das Handling des Pferdes während der Inhalation erleichtern.

\section{Literatur}

Ammann V. J., Vrins A. A., Lavoie J.-P. (1998) Effects of inhaled beclomethasone dipropionate on respiratory function in horses with chronic obstructive pulmonary disease (COPD). Equine Vet. J. 30, 152-157

Couëtil L. L., Hoffman A. M., Hodgson J., Buechner-Maxwell V., Viel L., Wood J. L., Lavoie J. P. (2007) Inflammatory airway disease of horses. J. Vet. Intern. Med. 21, 356-361

Couëtil L. L., Art T., de Moffarts B., Becker M., Mélotte D., Jaspar F., Bureau F., Lekeux P. (2006) Effect of beclomethasone dipropionate and dexame-thasone isonicotinate on lung function, bronchoalveolar lavage fluid cytology and transcription factor expression in airways of horses with recurrent airway obstruction. J. Vet. Intern. Med. 20, 399-406

Couëtil L. L., Chilcoat C. D., DeNicola D. B., Clark S. P., Glickman N. W., Glickman L. T. (2005) Randomized, controlled study of inhaled fluticasone propionate, oral administration of prednisone, and environmental management of horses with recurrent airway obstruction. Am. J. Vet. Res. 66, 1665-1674

Dauvillier J., Felippe M. J., Lunn D.P., Lavoie-Lamoureux A., Leclere M., Beauchamp G., Lavoie J. P. (2011) Effect of long-term fluticasone treatment on immune function in horses with heaves. J. Vet. Intern. Med. 25, 549-557

Derksen F. J., Olszewski M. A., Robinson N. E., Berney C., Hakala, J. E., Matson C. J. , Ruth D. T. (1999) Aerolized albuterol sulphate used as a bronchodilatator in horses with recurent airway obstruction. Am. J. Vet. Res. 60, 689-693

Duvivier D. H., Votion D., Vandenpu, S., Lekeux P. (1997) Aerosol therapy in the equine species. Vet. J. 154, 189-202

Fey K., Ohnesorge B. (2009) Alles Routine oder doch Herausforderung? Diagnostik und Therapie chronischer Atemwegserkrankungen beim Pferd. Vortragsheft, Fortbildungsveranstaltung Boehringer Ingelheim Vetmedica $\mathrm{GmbH}$.

Hoffman A. M., Foley M., Spendlove P. J. (2008) Respiratory medicine: new advances in inter-species aerosol delivery. Proc. Austr. Equine Science Symp. 2, 47

Hoffman A. M. (1997) Inhaled medications and bronchodilator usage in the horse. Vet. Clin. North. Am. Equine Pract. 13, 519-530

Kampmann C., Ohnesorge B., Venner M., Deegen. E. (2001) Budesamid-Aerosoltherapie und Lungenfunktion bei chronisch lungenkranken Pferden. Pferdeheilkunde 17, 155-160

Laan T. T., Bull S., van Nieuwstadt R. A., Fink-Gremmels J. (2006) The effect of aerosolized and intravenously administered clenbuterol and aeroso-lized fluticasone propionate on horses challenged with Aspergillus fumigatus antigen. Vet. Res. Commun. 30, 623-635

Leclere M., Lavoie J. P. (2014) Similarities between IAD and RAO. Proceedings of the 2nd Havemeyer Workshop on IAD, Cabourg (Frankreich), 87-89.

Niedermaier G., Gehlen H. (2009) Möglichkeiten der Inhalationstherapie zur Behandlung der chronisch obstruktiven Bronchitis des Pferdes. Pferdeheilkunde 25, 1-6

O'Connell E. J. (2003) Review of the unique properties of budesonide. Clin. Ther. 25 Suppl C, C42-60

Ohnesorge B., Trötschel C., Deegen E. (1998) Diagnostic value of capnography in horses with RAO. Proceedings 5th World Equine Vet. Assoc. Congress, 65-69

Pirie R. S. (2014) Recurrent airway obstruction: a review. Equine Vet J. $46,276-288$

Robinson N. E., Derksen F. J., Berney C., Goossens L. (1993) The airway response of horses with recurrent airway obstruction (heaves) to aerosol administration of ipratropiumbromide. Equine Vet. J. $25,299-303$

Robinson N. E., Derksen J. E., Jackson C. A, Peroni D., Gerber V. (2001) Management of heaves. Equine Vet. Educ. 13, 247-259

Rush B. R., Raub E. S., Thomsen M. M., Davis E. G., Matson C. J., Hakala J. E. (2000) Pulmonary function and adrenal gland suppression with incremental doses of aerosolized beclomethasone dipropionate in horses with recurrent airway obstruction. J. Am. Vet. Med. Assoc. 217, 359-364

Votion D., Ghafir Y., Munsters K., Duvivier D. H., Art T., Lekeux P. (1997) Aerosol deposition in equine lungs following ultrasonic nebulisation versus jet aerosol delivery system. Equine Vet. J. 29, 388-393 